\title{
Comparison of Body, Auricular, and Abdominal Acupuncture Treatments for Insomnia Differentiated as Internal Harassment of Phlegm-Heat Syndrome: An Orthogonal Design
}

\author{
Yue Jiao, ${ }^{1}$ Ying Han, ${ }^{1}$ Xin Li, ${ }_{1}^{1}$ Yi-gong Fang, ${ }^{1}$ Zhao-hui Liu, ${ }^{1}$ Wen-na Zhou, ${ }^{1}$ Jin-cao Zhou, \\ Zhong-chao Wu, ${ }^{1}$ Jin-hong Yang, ${ }^{1}$ Shao-yuan Li, ${ }^{1}$ Fan-ying Meng, ${ }^{2}$ and Wei-wei $\mathrm{Xu}^{3}$ \\ ${ }^{1}$ Institution of Acupuncture and Moxibustion, China Academy of Chinese Medical Sciences, Beijing 100700, China \\ ${ }^{2}$ TCM Hospital of Mentougou District, Beijing 102300, China \\ ${ }^{3}$ Medical College of Xiamen University, Xiamen 361000, China \\ Correspondence should be addressed to Yue Jiao; jiaoyue417@gmail.com
}

Received 25 August 2015; Revised 23 October 2015; Accepted 25 October 2015

Academic Editor: Gerhard Litscher

Copyright (c) 2015 Yue Jiao et al. This is an open access article distributed under the Creative Commons Attribution License, which permits unrestricted use, distribution, and reproduction in any medium, provided the original work is properly cited.

Objective. To identify the optimum treatment protocol for insomnia among auricular, body, and abdominal needling methods. Methods. A three-factor (3 needling protocols) and three-level experimental scheme was designed based on orthogonal method. 54 patients of insomnia differentiated as internal harassment of phlegm-heat syndrome were given two courses of acupuncture treatment (each with 20 times of acupuncture). The therapeutic effects were evaluated by comparing the Pittsburgh sleep quality index (PSQI), Hamilton Depression Scale (HAMD) scores, and Hamilton Anxiety Scale (HAMA) scores of patients before treatment, after one course of treatment, and after two courses of treatment as well as one month after treatment. Results. Body, auricular, and abdominal acupuncture treatments all alleviated symptoms of insomnia, depression, and anxiety, but body and auricular acupuncture had stronger therapeutic effects. Conclusions. Body acupuncture at basic points shall be given priority in protocol selection for insomnia. The second-best choice is auricular acupuncture with basic points combined with points based on Traditional Chinese Medicine (TCM) theories. Abdominal needling with very quick effect can be an alternative protocol with basic points combined with syndrome differentiation points.

\section{Introduction}

Insomnia is a sleep disorder that includes trouble falling asleep, staying asleep, or waking too early, resulting in daytime impairment [1]. About $30 \%$ of adults report at least one of the symptoms of insomnia. When daytime impairment is added as a criterion, the prevalence is about 10\% [2]. Longterm insomnia relates to body and mental health impairment, multivisceral dysfunction, and immune decline, as well as depression, anxiety, and other mental diseases [3]. Prevalence is higher among women [4], and, due to their physical and psychological peculiarity, women may easily get depression and anxiety as comorbidities of insomnia.

The sleep-wake cycle is a very complicated process, which involves central and peripheral nervous systems as well as the endocrine system $[5,6]$. Modern medicine has not possessed a complete and clear understanding of the pathogenesis so far, whereas it is commonly accepted that pathological changes occur in the anatomical structure of the sleepwake cycle, including inhibitory nucleus and arousal nucleus and the imbalance of corresponding neurotransmitters. In addition, endocrine regulation is in close relationship with the sleep-wake rhythm, for hormones like melatonin have been confirmed to have a curative effect on insomnia. To put it in a simple way, the pathogenesis manifests either as a weakening of the inhibitory function or as an enhancement of the arousal function.

From a Traditional Chinese Medicine (TCM) perspective, the pathogenesis of insomnia is intricate, involving disharmony of Zang and Fu organs (viscera) and disorder of Wei and Ying Qi (defensive qi and nutrient qi) as well as the Shen (spirit) disturbance of Zang organs. In brief, the pathogenesis 
of insomnia is regarded as imbalance between Yin and Yang, particularly a result of excessive Yang and deficient Yin, which is quite similar to the general understanding of western medicine.

Acupuncture is a simple but useful treatment for insomnia, with a success rate of around 90\% [7]. An explanation of modern medicine is that acupuncture can improve the neurotransmitter balance in the central nervous system. For instance, acupuncture increases the contents of $\gamma$-amino butyric acid (GABA) and Serotonin in the brain [8-10] and thus improves sleep quality. Improvement may be also due to the endocrine system, for example, the nocturnal increase in endogenous melatonin secretion [11]. Acupuncture treatment has several forms besides body needling, one group of which is micro needling system such as auricular and abdominal needling. Mechanisms underlying auricular and abdominal acupuncture are quite identical to the mechanism of body needling, which is characterized by dredging meridians and collaterals, building up body resistance to pathogenic factors, balancing Yin and Yang, and regulating the functions of Zang and Fu organs.

The study selected patients with phlegm-heat syndrome in TCM diagnosis based on references to traditional syndrome differentiation, clinical experiences, and literature review. As the life rhythm speeds up, more and more people suffer from inadequate sleep and improper diet. Their syndrome manifests as phlegm-heat. In the perspective of TCM, the pathogenesis of insomnia is "deficiency in origin and excess in symptom" (Ben Xu Biao Shi), and in most cases it cooccurs with phlegm and dampness as well as blood stasis [12]. The symptoms of phlegm-heat syndrome are as follows [13]: restless sleep, irritation and upset, suppression in the chest and gastric fullness, bitter taste in the mouth and profuse phlegm, dizziness, red tongue, yellow and thick tongue coating, and rapid pulse or rapid with slippery pulse.

Acupuncture is a complex therapy, including crucial factors such as syndrome differentiation, acupoint combination, and needling method as well as manipulation. Most clinical studies of insomnia treatments use single or combined protocols, whose therapeutic effects vary as different methods and indicators are used. Among Randomized Controlled Trials (RCTs) of acupuncture treatment for insomnia published in recent 10 years, the most commonly reported methods are body needling, auricular needling, abdominal needling, and their combinations. Although different influences are reported due to variances of methods and combination of acupoints, the effective rates in these studies are all highly or equally reported; moreover, there are various methods to form control groups, but the horizontal comparison and superiority trials are scanty. Aiming to identify an optimum protocol for insomnia, based on RCTs in China and abroad in recent ten years, this study chose the 3 highly evaluated protocols with different acupoints formula.

The orthogonal method is the best choice to design an experiment involving multivariate statistics. Its strengths lie in balanced distribution, calculation simplicity, and symmetrical comparability, so that it not only allows for effective experimental results with minimum time and cases of illness in clinical study, but also is capable of showing clearly interaction among factors and superiority among levels of each factor.

This study is designed as an orthogonal test to evaluate the effects of three acupuncture treatments on 54 patients with insomnia differentiated from internal harassment of phlegmheat syndrome.

\section{Materials and Methods}

2.1. Data Collection. 54 patients (45 females and 9 males) suffering from primary insomnia diagnosed by Diagnostic and Statistical Manual of Mental Disorders, Fourth Edition [1], and differentiated from phlegm-heat syndrome, all aged between 26 and 60 years (with the average age of $43.9 \pm$ 9.9 years), were enrolled in the study and treated in the Acupuncture Hospital of China Academy of Chinese Medical Sciences from May 2012 to May 2013.

2.2. Inclusion and Exclusion Criteria. Inclusion criteria were as follows:

(1) Primary insomnia patients diagnosed in accordance with DSM-IV, as mentioned above.

(2) Patients with primary insomnia differentiated from phlegm-heat syndrome, diagnosed in accordance with Terminology of Clinical diagnosis and treatment of TCM as mentioned above.

(3) Patients aged between 20 and 60 years (including 20and 60-year olds).

(4) The total score of Pittsburgh sleep quality index (PSQI) [14] $>7$.

(5) The score of Hamilton Depression Scale (HAMD) [15] $<20$.

(6) The score of Hamilton Anxiety Scale (HAMA) [16] < 14.

(7) Patients who signed the informed consent.

Exclusion criteria were as follows:

(1) Pregnancy and lactation.

(2) Patients with cardiovascular, lung, liver, kidney, or hemopoietic system diseases in late stages.

(3) Patients with mental diseases.

(4) Patients accepting related medication or other treatments.

(5) Severe adverse reactions to acupuncture (e.g., fainting during acupuncture).

\subsection{Method}

2.3.1. Grouping. The $\mathrm{L}_{9}\left(3^{4}\right)$ orthogonal array (Table 1 ) was used in the study. Patients were randomly assigned to nine protocols in a single-blinded study. In Table 1, alphabets A, $\mathrm{B}$, and $\mathrm{C}$ denote different treatment protocols and values 1 , 2 , and 3 indicate three different levels; alphabet $\mathrm{D}$ denotes the error term, which is excluded in the study. The details of protocols and levels employed in this paper are shown in Table 2 . 
TABLE 1: $\mathrm{L}_{9}\left(3^{4}\right)$ orthogonal array.

\begin{tabular}{lllll}
\hline \multirow{2}{*}{ Patient number } & \multicolumn{4}{c}{ Column number } \\
\hline 1 & A & B & C & D \\
2 & 1 & 1 & 1 & 1 \\
3 & 1 & 2 & 2 & 2 \\
4 & 1 & 3 & 3 & 3 \\
5 & 2 & 1 & 2 & 3 \\
6 & 2 & 2 & 3 & 1 \\
7 & 2 & 3 & 1 & 2 \\
8 & 3 & 1 & 3 & 2 \\
9 & 3 & 2 & 1 & 3 \\
\hline
\end{tabular}

TABLE 2: Designs for protocols and levels.

\begin{tabular}{ll}
\hline Protocols & Levels \\
\hline $\begin{array}{l}\text { A: body } \\
\text { acupuncture }\end{array}$ & $\begin{array}{l}\text { Level 1: 0 (body acupuncture not applied) } \\
\text { Level 3: basic acupoints } \\
\text { acupoints }\end{array}$ \\
\hline & Level 1: basic acupoints \\
B: auricular & Level 2: 0 (auricular acupuncture not applied) \\
acupuncture & Level 3: basic points + acupoints based on \\
& TCM theories \\
\hline & Level 1: basic acupoints \\
C: abdominal & Level 2: basic points + syndrome differentiation \\
acupuncture & acupoints \\
& Level 3: 0 (abdominal acupuncture not applied)
\end{tabular}

2.3.2. Treatment. Eight Chinese-trained and licensed acupuncturists with a median of 12 years of experience (range: 5 to 20 years) provided study treatments in the clinics. Before the study, all acupuncturists made consensus on the protocol of point selection and needle manipulations.

(1) Body Acupuncture. The acupuncture point selections were based on Traditional Chinese Medicine meridian theory to treat insomnia. The points were made references to the evidence-based guidelines of clinical practice with acupuncture and moxibustion: insomnia [17] and approved by committee of acupuncturists of the study.

Acupoints. The basic acupoints are Zhaohai (KI 6), Shenmai (BL 62), Shenmen (HT 7), Yintang (EX-HN 3), Sishencong (EX-HN 1), and Anmian (Ex-HN 22).

Acupoints selected on the basis of syndrome differentiation (hereafter referred to as syndrome differentiation acupoints) are Fenglong (ST 40), Neiting (ST 44), and Quchi (LI 11).

All acupoints were selected bilaterally except Yintang (EX-HN 3).

Operation. 1 cun $(0.25 \times 25 \mathrm{~mm})$ and $1.5 \mathrm{cun}(0.25 \times 40 \mathrm{~mm})$ disposable needles were used in the treatment. Patients laid in the supine position as the treatment proceeded. Needles were inserted with lifting and thrusting as well as rotating methods till qi sensation was felt. Among acupoints, Zhaohai (KI 6) was stimulated by the reinforcing technique, while Shenmai (BL 62) and syndrome differentiation acupoints were stimulated by the reducing technique. The rest of the acupoints were stimulated by the neutral reinforcing and reducing technique.

All points were stimulated for 1 minute in every 15 minutes. Needles were retained for 30 minutes.

Frequency and Courses of Treatment. Frequency and courses of treatment were as follows: once every other day, 3 times per week, 10 times in each course, and 2 consecutive courses in total.

(2) Auricular Acupuncture. The auricular point selections were based on TCM theory. The points were also made references to evidence-based guidelines of clinical practice with acupuncture and moxibustion: insomnia [17] and were approved by committee of acupuncturists of the study.

Acupoints. We used auricular acupoints as suggested in the nomenclature and location of auricular points [18].

The basic acupoints are Shenmen (TF4), Occiput (AT3), Chuiqian (LO4), and Subcortex (AT4).

Acupoints based on TCM theory were Spleen (CO13), Stomach (CO4), Heart (CO15), and Liver (CO12).

Acupoints were selected unilaterally at a time, and alternated every other day.

Operation 1 cun $(0.25 \times 25 \mathrm{~mm})$ disposable needles were used in the treatment. Patients took the supine position. After routine disinfection of acupoints, the needle was inserted and manipulated moderately without penetrating the skin.

The frequency and courses of treatment were the same as those of the body acupuncture.

(3) Abdominal Acupuncture. The abdominal acupuncture was guided by TCM theory and as one of holographic acupuncture methods. We used abdominal acupoints as suggested in the Abdominal Acupuncture [19].

Acupoints. Basic formula is as follows: Zhongwan (RN 12), Xiawan (RN10), Qihai (RN 6), and Guanyuan (RN 4); Shangqu (KI 17) (bilateral), Huaroumen (ST 24) (bilateral), Wailing (ST26) (bilateral), and Pinggan (extra point) (Bilateral).

Syndrome differentiation acupoints were Daheng (SP 15) (bilateral), Xiere (extra point) (bilateral).

Location of Pinggan was 0.7 cun outside and 0.3 cun above Huaroumen (ST 24); Xiere was 3 cun outside of Qihai (RN 6).

Operation. 1 cun $(0.22 \times 25 \mathrm{~mm})$ and 1.5 cun $(0.25 \times 40 \mathrm{~mm})$ disposable needles were used in the treatment. Patients took the supine position. After routine disinfection of acupoints, needles were vertically inserted into the skin according to the following sequence: from upward to downward, from inside to outside. 
TABLE 3: Baseline data of patients with insomnia.

\begin{tabular}{lcccccccc}
\hline Group & Cases & M & F & Age (years) & Duration of disease (years) & PSQI & HAMD & HAMA \\
\hline 1 & 6 & 1 & 5 & $43.83 \pm 11.05$ & $3.42 \pm 3.58$ & $13.67 \pm 3.01$ & $10.67 \pm 4.03$ & $7.83 \pm 4.17$ \\
2 & 6 & 1 & 5 & $44.17 \pm 10.67$ & $2.33 \pm 3.12$ & $11.67 \pm 3.93$ & $10.50 \pm 2.43$ & $7.83 \pm 3.13$ \\
3 & 6 & 1 & 5 & $40.00 \pm 15.87$ & $1.58 \pm 2.10$ & $14.83 \pm 1.60$ & $12.00 \pm 5.10$ & $8.33 \pm 3.50$ \\
4 & 6 & 1 & 5 & $44.00 \pm 14.25$ & $5.21 \pm 7.46$ & $13.33 \pm 4.18$ & $11.50 \pm 6.63$ & $8.50 \pm 3.83$ \\
5 & 6 & 1 & 5 & $41.67 \pm 7.76$ & $4.25 \pm 5.49$ & $12.33 \pm 3.14$ & $13.50 \pm 4.64$ & $10.50 \pm 5.21$ \\
6 & 6 & 1 & 5 & $43.83 \pm 13.00$ & $7.75 \pm 7.07$ & $12.00 \pm 3.29$ & $9.17 \pm 2.32$ & $8.33 \pm 3.27$ \\
7 & 6 & 1 & 5 & $37.17 \pm 10.98$ & $2.58 \pm 3.77$ & $9.83 \pm 2.86$ & $10.67 \pm 3.56$ & $9.83 \pm 3.25$ \\
8 & 6 & 1 & 5 & $41.50 \pm 12.18$ & $2.50 \pm 2.56$ & $11.50 \pm 3.45$ & $8.00 \pm 4.90$ & $6.67 \pm 5.24$ \\
9 & 6 & 1 & 5 & $41.50 \pm 10.41$ & $1.19 \pm 1.58$ & $14.17 \pm 3.43$ & $11.50 \pm 5.24$ & $8.17 \pm 3.66$ \\
Sum & 54 & 9 & 45 & $41.96 \pm 11.80$ & $4.26 \pm 5.34$ & $12.59 \pm 3.21$ & $10.83 \pm 4.32$ & $8.44 \pm 3.92$ \\
\hline
\end{tabular}

Needles were applied at Zhongwan (RN 12), Xiawan (RN10), Qihai (RN 6), and Guanyuan (RN 4), touching the linea alba of Ren meridian (fascia layer) and after needles were inserted at Zhongwan (RN 12), Xiawan (RN10), and Qihai (RN 6), the direction of needle tips was adjusted to be inserted obliquely at an angle of $45^{\circ}$ along the Ren Meridian, while a needle was inserted vertically at Guanyuan (RN 4).

After a needle was applied at Shangqu (KI 17), the direction of the needle tip was adjusted to move obliquely downward at an angle of $45^{\circ}$ along the kidney meridian to the depth of the fat layer.

Needles were inserted vertically at Huaroumen (ST 24), Wailing (ST26), Pinggan, Daheng (SP 15), and Xiere, to the fat layer at Huaroumen (ST 24), Wailing (ST 26), and Daheng (SP 15), to the muscle layer at Pinggan and Xier.

The frequency and courses of treatment were the same as those of the body acupuncture.

Patients were kept warm during treatment.

2.4. Clinical Outcomes. Sleep quality of patients was evaluated with PSQI scores before the treatment and after 1 course and 2 courses of treatment and 1 month after treatment. Similarly, depression was evaluated with HAMD scores and anxiety with HAMA scores.

2.5. Statistical Analysis. SPSS17.0 was used to analyze data. A descriptive analysis was performed first, and then was followed by a visual calculation in accordance with requests of orthogonal test. The $R$ value indicated the influences of different protocols; that is to say, a higher score of $R$ meant the protocol was more effective.

\section{Results}

3.1. General Data. There was no statistically significant difference between the groups in terms of age, duration of disease, and scores of PSQI, HAMD, and HAMA. The average age, duration of disease, and PSQI, HAMD, and HAMA scores were $41.96 \pm 11.80$ (years), $4.26 \pm 5.3$ (years), and $12.59 \pm$ $3.21,10.83 \pm 4.32$, and $8.44 \pm 3.92$, respectively (Table 3).

3.2. PSQI Scoring. The $R$ values symbolizing the range of PSQI score in orthogonal test were shown in Figure 1. As time

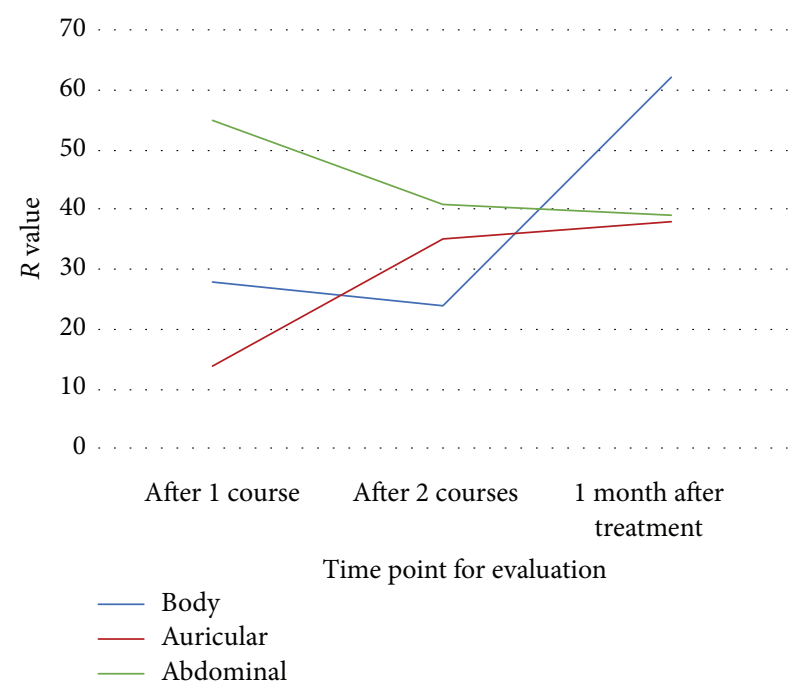

FIGURE 1: Influences on PSQI scores of different protocols at each time point.

went by, auricular acupuncture demonstrated an accelerating trend on PSQI score reduction, while abdominal acupuncture showed a decelerating trend. Body acupuncture presented an impressive promotion after treatment rather than during the treatment (Figure 1).

Table 4 compares the PSQI score reduction from three levels of three protocols. It indicates that A2 basic acupoints, B3 basic points + acupoints based on TCM theories, and $\mathrm{C} 2$ basic + syndrome differentiation acupoints had stronger influences on the descending trend of PSQI scores, which meant continuous improvement of sleep quality (Table 4).

3.3. HAMD Scoring. The $R$ values symbolizing the range of HAMD score in orthogonal test were shown in Figure 2. As time went by, auricular acupuncture demonstrated first a sharply decelerating and then accelerating trend on HAMD score reduction, while abdominal acupuncture showed an accelerating and then decelerating trend. Body acupuncture presented an impressive promotion especially after the treatment. 
TABLE 4: PSQI comparison among three levels of each protocol (factor).

\begin{tabular}{lccc}
\hline Group & After 1 course & After 2 courses & 1 month after treatment \\
\hline A1 & 79 & 116 & 111 \\
A2 & 87 & 104 & 134 \\
A3 & 75 & 115 & 81 \\
B1 & 75 & 116 & 101 \\
B2 & 81 & 104 & 92 \\
B3 & 85 & 115 & 133 \\
C1 & 52 & 98 & 91 \\
C2 & 109 & 132 & 125 \\
C3 & 80 & 105 & 110 \\
\hline
\end{tabular}

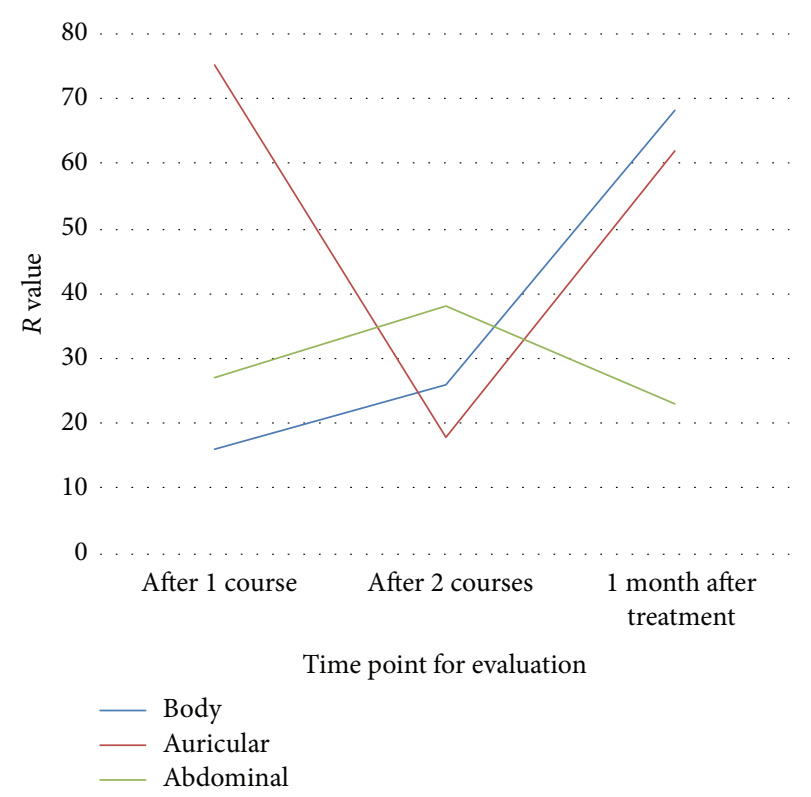

Figure 2: Influences on HAMD scores of different protocols at each time point.

Table 5 compares the HAMD score reduction from three levels of three protocols. It indicates that A2 basic acupoints, B3 basic points + acupoints based on TCM theories, and $\mathrm{C} 2$ basic + syndrome differentiation acupoints had stronger influences on the descending trend of HAMD score, which meant improvement in depression (Table 5).

3.4. HAMA Scoring. The $R$ values symbolizing the range of HAMA score in orthogonal test were shown in Figure 3. As time went by, auricular acupuncture demonstrated a slightly decelerating and then accelerating trend on HAMD score reduction, while abdominal acupuncture showed a decelerating trend, whereas body acupuncture presented an accelerating trend.

Table 6 compares the HAMD score reduction from three levels of three protocols. It indicates that A2 basic acupoints, $\mathrm{B} 1$ basic acupoints, and $\mathrm{C} 2$ basic + syndrome differentiation acupoints had stronger influences on the descending trend of HAMA score, which meant improvement in anxiety (Table 5).
TABLE 5: HAMD comparison among three levels of each protocol (factor).

\begin{tabular}{lccc}
\hline Group & After 1 course & After 2 courses & 1 month after treatment \\
\hline A1 & 57 & 90 & 85 \\
A2 & 66 & 93 & 109 \\
A3 & 50 & 67 & 41 \\
B1 & 40 & 82 & 57 \\
B2 & 29 & 75 & 59 \\
B3 & 104 & 93 & 119 \\
C1 & 45 & 92 & 66 \\
C2 & 72 & 98 & 88 \\
C3 & 56 & 60 & 81 \\
\hline
\end{tabular}

TABLE 6: HAMA comparison among three levels of each protocol (factor).

\begin{tabular}{lccc}
\hline Group & After 1 course & After 2 courses & 1 month after treatment \\
\hline A1 & 33 & 76 & 78 \\
A2 & 48 & 72 & 95 \\
A3 & 46 & 47 & 58 \\
B1 & 36 & 73 & 94 \\
B2 & 34 & 59 & 53 \\
B3 & 57 & 63 & 84 \\
C1 & 47 & 73 & 76 \\
C2 & 49 & 67 & 79 \\
C3 & 31 & 55 & 76 \\
\hline
\end{tabular}

\section{Discussion}

The study used orthogonal design, an experimental design used to test the comparative effectiveness of 3 intervention components (protocols), each of which took on 3 variants (levels) in this study, because it allowed testing the effectiveness of body, auricular, and abdominal acupuncture simultaneously in a single study with far fewer experimental units than it would take to exhaust all possible intervention combinations [20]. To design and implement this technique, orthogonal array would first be chosen to fit the study purpose. Since 3 kinds of acupuncture were tested for interaction, and point selection was the second consideration, then it would be structured to the 3 factors (the number of columns in an array) and 3 levels (the maximum number of values that can be taken on by any single factor) of the array. With respect to suitable orthogonal array selection (orthogonal arrays are most often named following the pattern $\mathrm{L}_{\text {Runs }}$ (Levels ${ }^{\text {Factors }}$ )), the rule is to find one with the smallest number of runs (the number of test cases) [21]. Since there was no orthogonal array of 3 factors and 3 levels, then $\mathrm{L}_{9}\left(3^{4}\right)$ orthogonal array was most suitable in the study.

Among the three protocols, body needling had steadily accelerating effectiveness on improvement of insomnia, depression, and anxiety in both the treatment and the followup period. Therefore it was the most recommended therapy and the best level was to use basic acupoints for treatment. The mechanism of body needling treatment for insomnia in TCM included regulating Yin Qiao and Yang Qiao meridians 


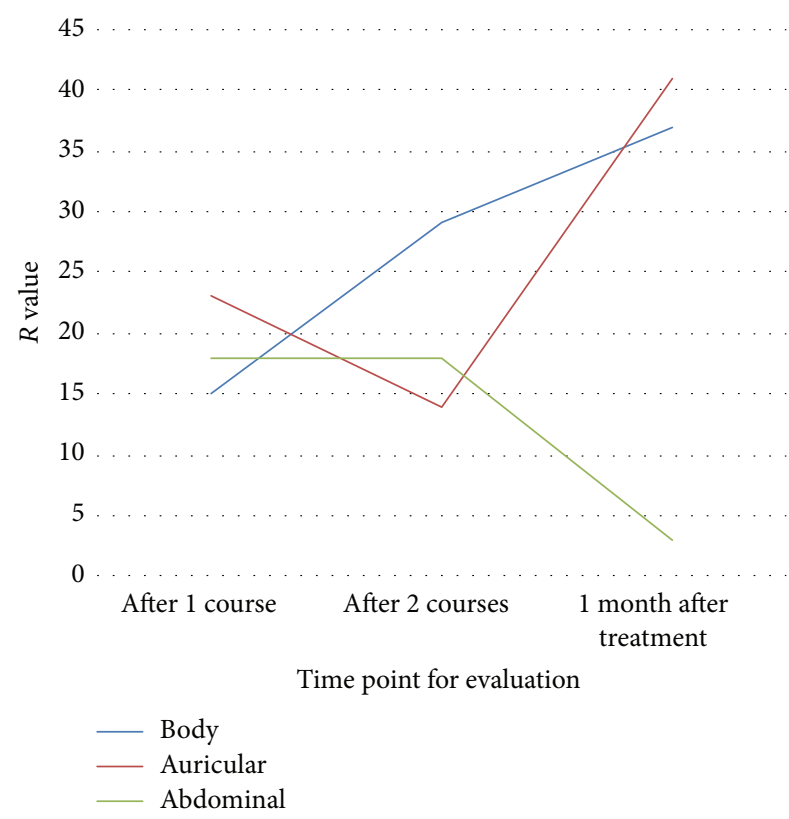

FIgURE 3: Influence on HAMA scores of different protocols at each time point.

with points of Zhaohai (KI 6) and Shenmai (BL 62) to balance Yin and Yang based on the Yin-yang theory and Ying-wei theory; tonifying the heart and calming the mind with Shenmen (HT 7), Yintang (EX-HN 3), Sishencong (EXHN 1), and Anmian (Ex-HN 22) based on the shen- (spirit-) dominating-sleep theory. The basic points alone were able to adjust the function of the whole body, so that was enough to take effect on insomnia patients with internal harassment of phlegm-heat syndrome. However, further studies are needed to explore suitable protocols for insomnia patients with other syndromes.

In TCM, heart is the master of five viscera including heart, lung, spleen, liver, and kidney. "Shen of heart," dominating mental activity, is self-consciousness and unique human ability. Their function however quite resembles cerebrum. If shen calms down, humans fall asleep, while when shen activates, they wake up. Moreover, sleep follows daily biological rhythm and shows as a sign of balance of Yin and Yang. In TCM, if wei qi flourishes in Yang qiao meridian, eyes open and consciousness is regained, while if wei qi flourished in Yin qiao meridian, eyes close and sleep will come. According to meridian circulation, Yin qiao and Yang qiao meridians confluence at inner canthus and then enter the brain. In other words, they are both crucial routes related to sleep. Thus it can be seen that TCM theory considers the fact that sleep is closely linked with brain function, so that most of acupoints selected to treat insomnia locate on the head. In TCM, the insomnia treating principle lies in balancing Yin and Yang. And fMRI can be taken as one of parameters of insomnia regulations by acupuncture. Gao et al. [22] reported that, in sleep deprivation, an imbalance occurs; acupuncture stood for a homeostatic force to renormalize the Yin and Yang; biphasic regulation effects of acupuncture, the salience network, composed of the anterior insular cortex and anterior cingulate (ACC), a unique interoceptive autonomic circuit, might indicate the mechanism underlying acupuncture in restoring sleep deprivation. Effects of acupuncture were closing to launch homeostatic regulation [23, 24].

The auricular needling also had a clear therapeutic effect on insomnia, depression, and anxiety. Unlike body needling, its efficacy in treating insomnia increased continuously, while, in ameliorating depression and anxiety, the increase got decelerated in the treatment period while it was accelerated during follow-up period. From the respective of TCM, the auricle had close relationships with both meridians and viscera. Holographic medicine and modern medicine have also confirmed the somatotopic function of specific regions of the ear. Various nerves including the spinal and cranial nerves are distributed at the auricles, particularly in the triangular fossa and the concha region. Among those nerves, the vagus nerve is a mixed nerve composed of about $80 \%$ afferent fibers. The concha area has a rich distribution of the vagus nerve. Stimulating points in this region with needles can adjust functions of the viscera and the central nervous system, as well as the autonomous system [25-27]. It was speculated that the insomnia regulating effects of vagus nerve stimulation were partially attributed to the projection of afferent fibers to the nucleus tractus solitarius, which was further connected directly and indirectly with brain structures [28] including the locus coeruleus, cerebral cortex, hippocampus, thalamus, and cerebellum [29]. Just yet, some of these brain regions were also believed to be involved in the pathogenesis of sleep disorder [30-32], which built a basis for treatment with vagus nerve stimulation [29]. Moreover, excitation of the parasympathetic nerve, which was part of the vagus nerve, stimulated the pineal gland to secrete melatonin [33-37]. Insomnia closely related to functional decrease of central melatonin; mutual promotion between excitation of parasympathetic nerve and melatonin secretion became a basis of treating insomnia [38-41]. It might partly explain the therapeutic effect of auricular acupuncture. Furthermore, basic points like Occiput (AT3), Chuiqian (LO4), and Subcortex (AT4) were mainly used as corresponding points of the brain. Stimulation at these points and Shenmen (TF4) calms the mind. Points based on TCM theory such as spleen (CO13), stomach (CO4), heart (CO15), and liver (CO12) can regulate viscera functions to improve phlegm-heat symptoms, and since they are all located in the concha region, these points can improve sleep quality by stimulating the vagus nerve. Besides, the stimulation of auricular needling was stronger than the other two protocols, so it may take effect very quickly, especially for the treatment of anxiety and irritation, and it might explain why it had rapid and solid effect in depression and anxiety.

Brain imaging tools have been used to investigate the fMRI signal change evoked by transcutaneous vagus nerve stimulation recently [42]. Kraus et al. [42] found that robust tVNS can induce fMRI signal decreases in limbic brain areas, including the amygdala, hippocampus, parahippocampal gyrus, and middle and superior temporal gyrus, as well as an fMRI signal increase in the insula, precentral gyrus, and thalamus. As mentioned above, these were all important structures related to sleep-wake cycle. In recent study of tVNS 
treating major depressive disorder, by the help of fMRI, Fang et al. [43] found that significant functional connectivity (FC) between default mode network (DMN) and brain regions such as the parahippocampus, ACC, and medial temporal areas. These results suggested that the modulation of tVNS was not targeted at one particular region but it rather influenced brain region networks associated with emotion/affect regulation. Since insomnia also related intimately to depression and anxiety, it might provide a reference approach for treatment and a mechanism explanation.

Abdominal needling could improve insomnia, depression, and anxiety with a gradually decelerated increase. The abdominal region is where the viscera and meridians are located, including the Ren, kidney, spleen, and stomach as well as gallbladder and branch meridian of Du meridians. The abdominal needling helped to harmonize Yin and Yang and regulate meridians and viscera, which corresponded to the pathogenesis of insomnia: imbalance of Yin-Yang and disorder of viscera function. Furthermore, the abdominal needling demanded slight and shallow stimulation, with finer needles, which minimized pain compared with traditional needling techniques, so it was easily accepted by patients, particularly at the initial stage of the treatment. That might explain its remarkable effect at the beginning of treatment. Basic formula combined with syndrome differentiation points produced a stable and better effect. Among the points, Daheng (SP 14) and Xiere are located on and near the spleen meridian, which might strengthen the spleen's function of dispersing phlegm. Wang [44] applied abdominal needling treatment for depression; the author observed that, by means of fMRI-ReHo, patients suffered abnormal changes in several brain regions in resting-state, especially limbiccortical-striatal-pallidal-thalamic (LCSPT) circuit; however, after abdominal needling treatment, their function could be regulated through amelioration of blood oxygen and metabolism. The circuit and cortico-striato-thalamic-cortical loop (CSTC loop) overlapped quit a lot in the brain. The latter was known to regulate cortical arousal by controlling the effectiveness of the thalamic filter; this system regulated cortical arousal by filtering out sensory input for maintaining sleep or by allowing specific sensory input to the cortex for maintaining cortical arousal [30]. Therefore, one explanation for the fact that abdominal needling took effect on insomnia might lay in the circuit mentioned above.

It shall be noted that our study had several limitations. Firstly, the syndrome differentiation of patients in the study was internal harassment of phlegm-heat syndrome, so the conclusions shall not be applied to other syndromes. Secondly, though the study efficiently compared the therapeutic effects of three protocols and corresponding points selection with a $\mathrm{L}_{9}\left(3^{4}\right)$ table of orthogonal design, it did not examine the interaction effects between them. Therefore, the conclusions did not address the question of whether the protocols shall be used together or separately. Thirdly, both the auricular and abdominal needling showed a significant therapeutic effect at the beginning of treatment, but the increasing effect of auricular needling was unsteady for depression and anxiety and so was the increasing effect of abdominal needling. Differences between body needling and micro-needling system, as well as between different micro-needling systems, need further studies. And finally, the sample in this study was relatively small. The mechanism needs to be researched with more diversified patients and sophisticated experimental designs from both the TCM and modern medicine perspectives.

\section{Conclusions}

The following conclusions can be drawn from this study:

(1) Body, auricular, and abdominal needling all improved insomnia and depression as well as anxiety for patients with internal harassment of phlegm-heat syndrome, with the first two protocols being more effective.

(2) Among the three protocols, body needling is firstly recommended for it consistently improved insomnia and depression as well as anxiety, and the therapeutic effect kept improving as time went by. The recommended points are basic ones.

(3) Auricular needling improved insomnia with a sustainably growing trend, but, for depression and anxiety, within the treatment period, the increasing is decelerated than that during follow-up period. The recommended points are basic points combined with points based on TCM theory.

(4) Abdominal needling improves insomnia with a decelerated increase, and, for depression and anxiety, the increase is first accelerated within treatment period, then decelerated during follow-up period. The recommended points are basic ones combined with syndrome differentiation points.

\section{Conflict of Interests}

The authors declare that they have no conflict of interests.

\section{Acknowledgments}

This research was supported by the Independent Topic Project of China Academy of Chinese Medical Sciences (ZZ12007) and the National Natural Science Foundation of China (81473780). The Acupuncture Hospital affiliated to China Academy of Chinese Medical Science and the Institutional Ethics Committee of the China Academy of Chinese Medical Sciences are thanked for their invaluable contribution to the study. The authors would also like to thank the participants for volunteering their time to the study.

\section{References}

[1] American Psychiatric Association (APA), Diagnostic and Statistical Manual of Mental Disorders: DSM-IV-TR, American Psychiatric Publishing, 4th edition, 2000.

[2] T. Roth, "Insomnia: definition, prevalence, etiology, and consequences," Journal of Clinical Sleep Medicine, vol. 3, no. 5, pp. 7-10, 2007. 
[3] D. E. Ford and D. B. Kamerow, "Epidemiologic study of sleep disturbances and psychiatric disorders. An opportunity for prevention?" The Journal of the American Medical Association, vol. 262, no. 11, pp. 1479-1484, 1989.

[4] G. D. Mellinger, M. B. Balter, and E. H. Uhlenhuth, "Insomnia and its treatment: prevalence and correlates," Archives of General Psychiatry, vol. 42, no. 3, pp. 225-232, 1985.

[5] K. Spiegel, R. Leproult, and E. Van Cauter, "Impact of sleep debt on metabolic and endocrine function," The Lancet, vol. 354, no. 9188, pp. 1435-1439, 1999.

[6] K. Spiegel, E. Tasali, P. Penev, and E. Van Cauter, "Brief communication: sleep curtailment in healthy young men is associated with decreased leptin levels, elevated ghrelin levels, and increased hunger and appetite," Annals of Internal Medicine, vol. 141, no. 11, pp. 846-850, 2004.

[7] Y. Lin, "Acupuncture treatment for insomnia and acupuncture analgesia," Psychiatry and Clinical Neurosciences, vol. 49, no. 2, pp. 119-120, 1995.

[8] J. S. Han and L. Terenius, "Neurochemical basis of acupuncture analgesia," Annual Review of Pharmacology and Toxicology, vol. 22, pp. 193-220, 1982.

[9] J. S. Han, "Electroacupuncture: an alternative to antidepressants for treating affective diseases?" International Journal of Neuroscience, vol. 29, no. 1-2, pp. 79-92, 1986.

[10] M. Pi, S. H. Du, and Y. Zhang, "Study of therapeutic effect of acupuncture on insomnia and its effect on $\gamma$-amino butyric acid," Journal of Jiangxi College of Traditional Chinese Medicine, vol. 12, p. 160, 2000.

[11] D. W. Spence, L. Kayumov, A. Chen et al., "Acupuncture increases nocturnal melatonin secretion and reduces insomnia and anxiety: a preliminary report," Journal of Neuropsychiatry and Clinical Neurosciences, vol. 16, no. 1, pp. 19-28, 2004.

[12] X. Li, TCM Syndrome differentiation of primary insomnia [Ph.D. thesis], Beijing University of Chinese Medicine, Beijing, China, 2007 (Chinese).

[13] The State Bureau of Technology Supervision, Terminology of Clinical Diagnosis and Treatment of TCM: Syndromes, National Standard of People's Republic of China, China Standard Publishing House, China, 1997.

[14] D. J. Buysse, C. F. Reynolds III, T. H. Monk, S. R. Berman, and D. J. Kupfer, "The Pittsburgh sleep quality index: a new instrument for psychiatric practice and research," Psychiatry Research, vol. 28, no. 2, pp. 193-213, 1989.

[15] J. B. Williams, "A structured interview guide for the hamilton depression rating scale," Archives of General Psychiatry, vol. 45, no. 8, pp. 742-747, 1988.

[16] W. Maier, R. Buller, M. Philipp, and I. Heuser, "The Hamilton Anxiety Scale: reliability, validity and sensitivity to change in anxiety and depressive disorders," Journal of Affective Disorders, vol. 14, no. 1, pp. 61-68, 1988.

[17] China Association of Acupuncture-Moxibustion (CAAM), The Evidence-Based Guidelines of Clinical Practice with Acupuncture and Moxibustion: Insomnia, China Traditional Medicine Press, 1st edition, 2014.

[18] Standardization Administration of China, Nomenclature and Location of Auricular Points: National Standard of People's Republic of China, China Standard Publishing House, Beijing, China, 2008.

[19] Z. Y. Bo, Abdominal Acupuncture, Chinese Press of Traditional Chinese Medicine, Beijing, China, 2010.
[20] J. Zurovac and R. Brown, "Orthogonal Design: A Powerful Method for Comparative Effectiveness Research with Multiple Interventions," 2015, http://www.mathematica-mpr.com/ / media/publications/PDFs/health/orthogonaldesign_ib.pdf.

[21] J. M. Harell, "Orthogonal Array Testing Strategy (OATS) Technique," 2015, http://www.51testing.com/ddimg/uploadsoft/ 20090113/OATSEN.pdf.

[22] L. Gao, M. Zhang, H. H. Gong et al., "Differential activation patterns of fMRI in sleep-deprived brain: restoring effects of acupuncture," Evidence-Based Complementary and Alternative Medicine, vol. 2014, Article ID 465760, 7 pages, 2014.

[23] R. P. Dhond, N. Kettner, and V. Napadow, "Neuroimaging acupuncture effects in the human brain," Journal of Alternative and Complementary Medicine, vol. 13, no. 6, pp. 603-616, 2007.

[24] L. Bai and L. Lao, "Neurobiological foundations of acupuncture: the relevance and future prospect based on neuroimaging evidence," Evidence-Based Complementary and Alternative Medicine, vol. 2013, Article ID 812568, 9 pages, 2013.

[25] P.-J. Rong, J.-L. Fang, J. Kong et al., “Transcutaneous vagus nerve stimulation for the treatment of depression: a study protocol for a double blinded randomized clinical trial," $B M C$ Complementary and Alternative Medicine, vol. 12, no. 1, article 255, 2012.

[26] R. La Marca, M. Nedeljkovic, L. Yuan, A. Maercker, and U. Ehlert, "Effects of auricular electrical stimulation on vagal activity in healthy men: evidence from a three-armed randomized trial," Clinical Science, vol. 118, no. 8, pp. 537-546, 2010.

[27] R. Round, G. Litscher, and F. Bahr, "Auricular acupuncture with laser," Evidence-Based Complementary and Alternative Medicine, vol. 2013, Article ID 984763, 22 pages, 2013.

[28] A. Valdés-Cruz, V. M. Magdaleno-Madrigal, D. MartínezVargas et al., "Chronic stimulation of the cat vagus nerve: effect on sleep and behavior," Progress in Neuro-Psychopharmacology and Biological Psychiatry, vol. 26, no. 1, pp. 113-118, 2002.

[29] D. Vigier and P. Portalier, "Efferent projections of the area postrema demonstrated by autoradiography," Archives Italiennes de Biologie, vol. 117, no. 4, pp. 308-324, 1979.

[30] S. M. Stahl, Essential Psychopharmacology: Neuroscientific Basis and Practical Applications, Cambridge University Press, Cambridge, UK, 2007.

[31] C. B. Saper, T. E. Scammell, and J. Lu, "Hypothalamic regulation of sleep and circadian rhythms," Nature, vol. 437, no. 7063, pp. 1257-1263, 2005.

[32] H. Haas and P. Panula, "The role of histamine and the tuberomamillary nucleus in the nervous system," Nature Reviews Neuroscience, vol. 4, no. 2, pp. 121-130, 2003.

[33] G. C. Brainard, "Photic parameters that regulate the neuroendocrine system and influence behavior in humans and animals," Photodermatology, Photoimmunology \& Photomedicine, vol. 8, pp. 34-39, 1991.

[34] C. E. Remé, A. Wirz-Justice, and M. Terman, “The visual input stage of the mammalian circadian pacemaking system. I. Is there a clock in the mammalian eye?" Journal of Biological Rhythms, vol. 6, no. 1, pp. 5-29, 1991.

[35] J. E. Roberts, "Light and immunomodulation," Annals of the New York Academy of Sciences, vol. 917, pp. 435-445, 2000.

[36] T. Mutho, S. Shibata, H.-W. Korf, and H. Okamura, "Melatonin modulates the light-induced sympathoexcitation and vagal suppression with participation of the suprachiasmatic nucleus in mice," The Journal of Physiology, vol. 547, no. 1, pp. 317-332, 2003. 
[37] E. L. Haus and M. H. Smolensky, "Shift work and cancer risk: potential mechanistic roles of circadian disruption, light at night, and sleep deprivation," Sleep Medicine Reviews, vol. 17, no. 4, pp. 273-284, 2013.

[38] A. Brzezinski, "Melatonin in humans," The New England Journal of Medicine, vol. 336, no. 3, pp. 186-195, 1997.

[39] T. Roehrs and T. Roth, "Insomnia pharmacotherapy," Neurotherapeutics, vol. 9, no. 4, pp. 728-738, 2012.

[40] V. Srinivasan, S. R. Pandi-Perumal, I. Trahkt et al., "Melatonin and melatonergic drugs on sleep: possible mechanisms of action," International Journal of Neuroscience, vol. 119, no. 6, pp. 821-846, 2009.

[41] R. Mahlberg, T. Kienast, S. Hädel, J. O. Heidenreich, S. Schmitz, and D. Kunz, "Degree of pineal calcification (DOC) is associated with polysomnographic sleep measures in primary insomnia patients," Sleep Medicine, vol. 10, no. 4, pp. 439-445, 2009.

[42] T. Kraus, K. Hösl, O. Kiess, A. Schanze, J. Kornhuber, and C. Forster, "BOLD fMRI deactivation of limbic and temporal brain structures and mood enhancing effect by transcutaneous vagus nerve stimulation," Journal of Neural Transmission, vol. 114, no. 11, pp. 1485-1493, 2007.

[43] J. L. Fang, P. J. Rong, Y. Hong et al., “Transcutaneous vagus nerve stimulation modulates default mode network in major depressive disorder," Biological Psychiatry, 2015.

[44] B. W. Wang, fMRI study on the effect of Bo Shi abdominal acupuncture on women with depression [Ph.D. thesis], Guangzhou University of Chinese Medicine, Guangzhou, China, 2011. 


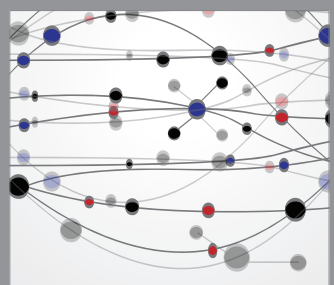

The Scientific World Journal
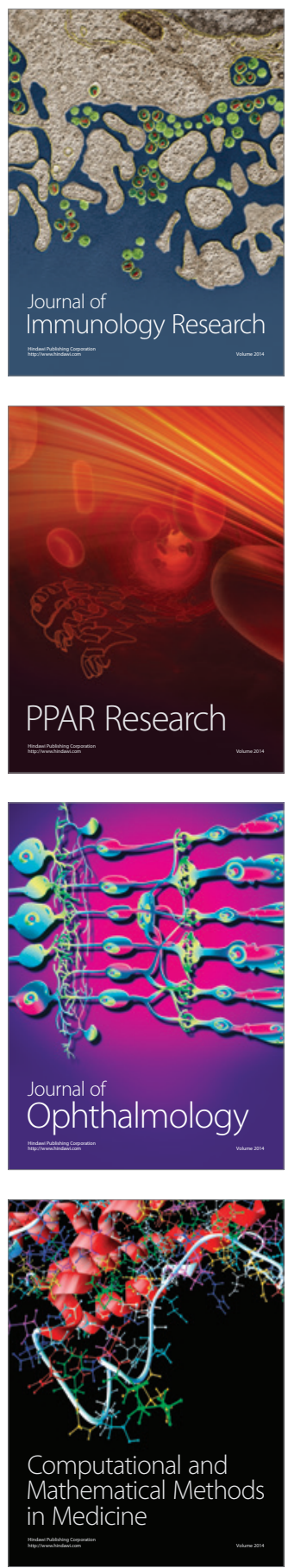

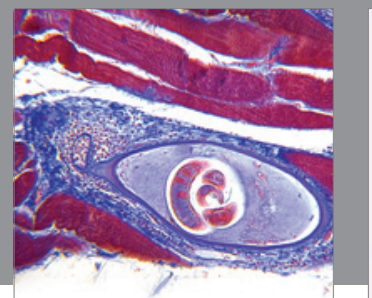

Gastroenterology

Research and Practice
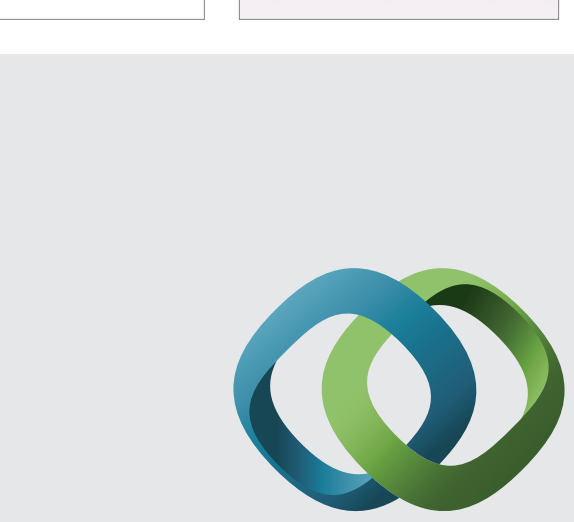

\section{Hindawi}

Submit your manuscripts at

http://www.hindawi.com
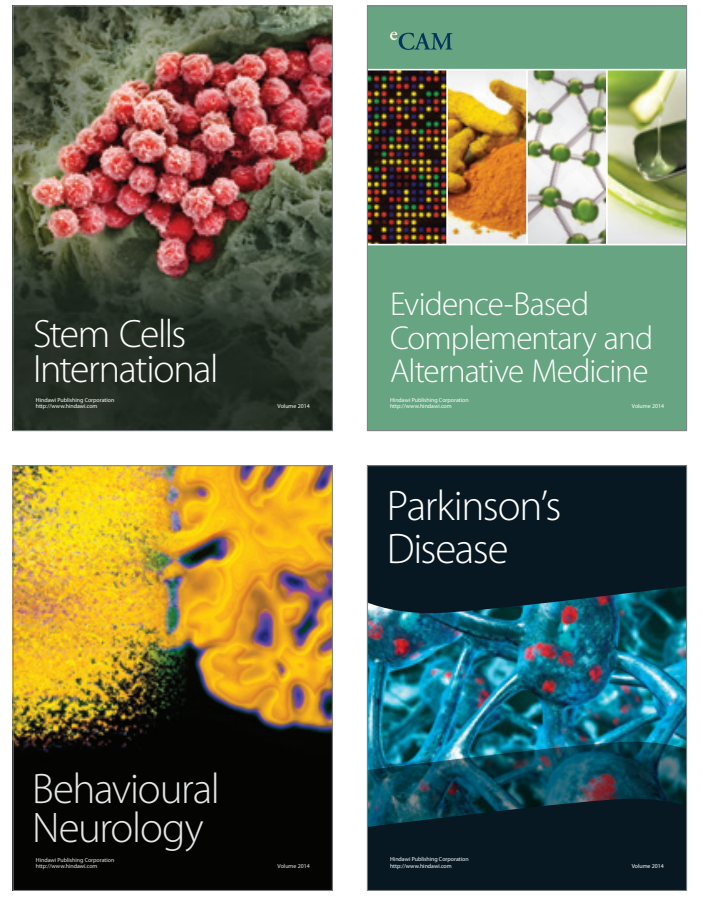
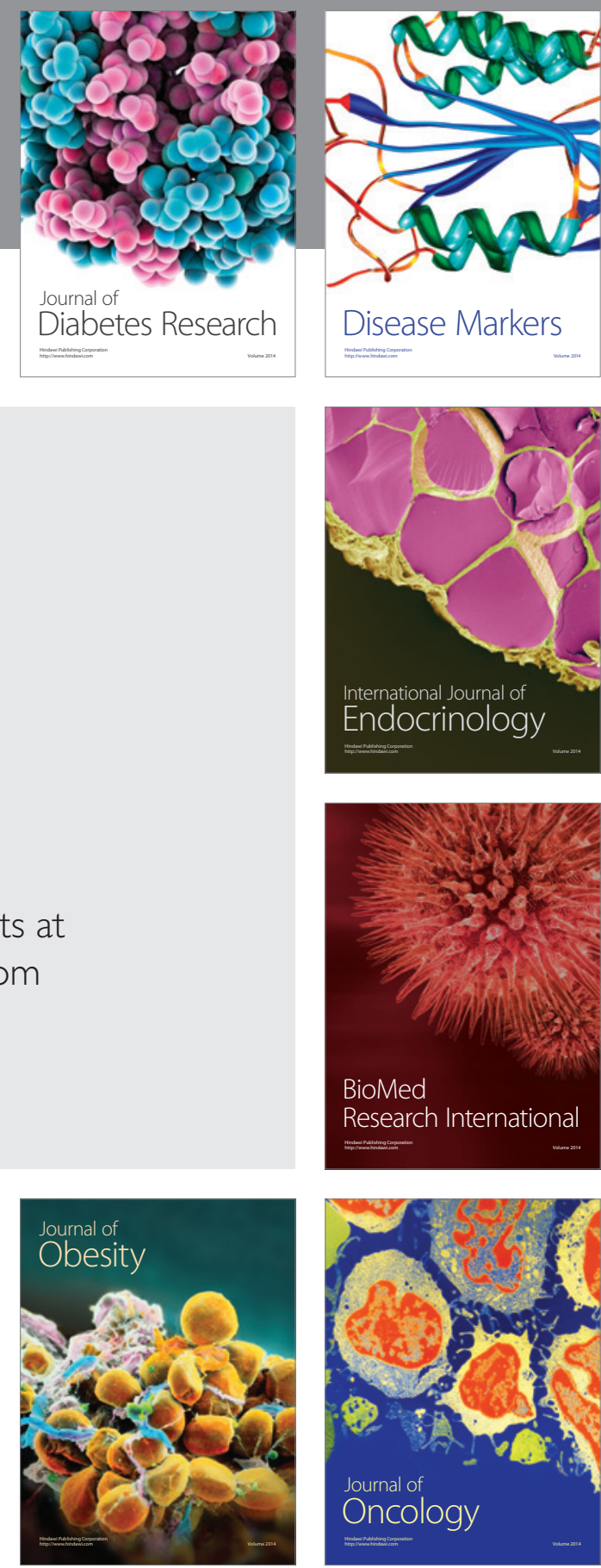

Disease Markers
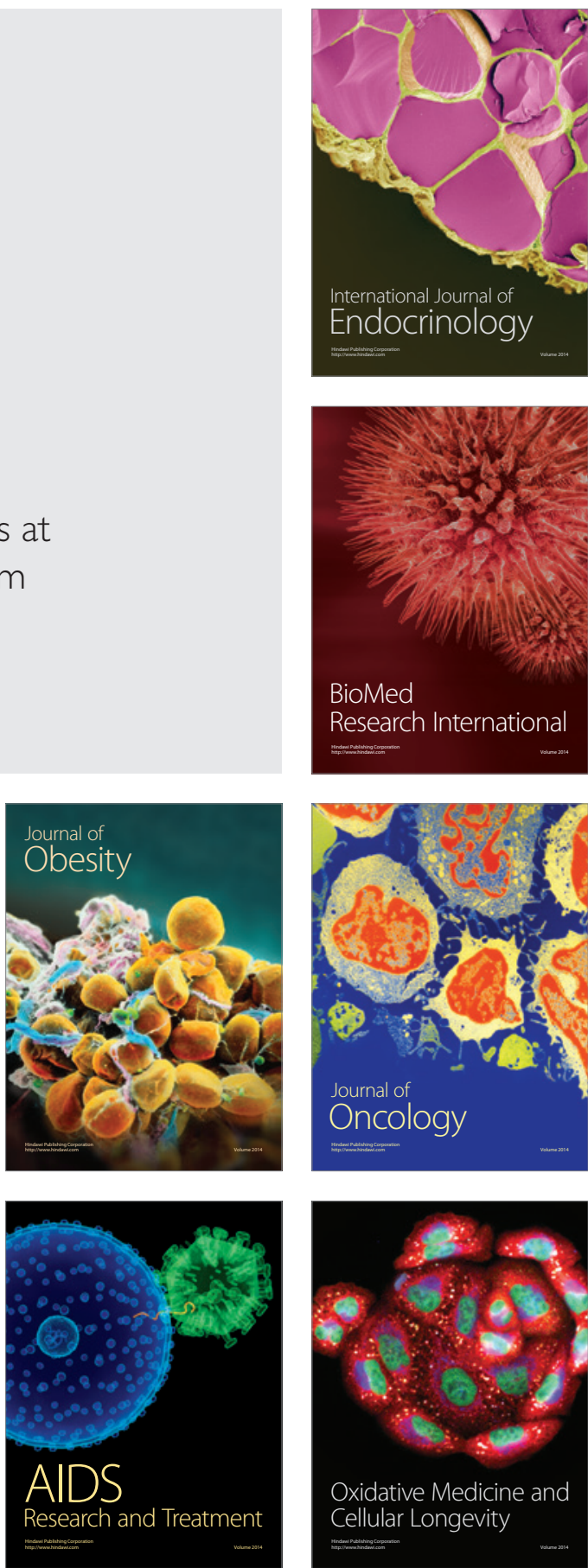